\title{
Weierstrass' Elliptic Function Solutions to the Autonomous Limit of the String Equation
}

\author{
Yoshikatsu Sasaki \\ Department of Mathematics, Hiroshima University, Higashi-Hiroshima, Japan \\ Email: sasakiyo@hiroshima-u.ac.jp
}

Received 4 January 2016; accepted 23 April 2016; published 30 April 2016

\begin{abstract}
In this article, we study the string equation of type $(2,2 n+1)$, which is derived from $2 \mathrm{D}$ gravity theory or the string theory. We consider the equation as a $2 n$-th order analogue of the first Painlev éequation, take the autonomous limit, and solve it concretely by use of the Weierstrass' elliptic function.
\end{abstract}

\section{Keywords}

Painlevé Hierarchy, String Equation, Elliptic Function

\section{Introduction}

\subsection{The String Equation of Type $(2,2 n+1)$}

Let $D$ or ${ }^{\prime}$ stand for the differentiation w.r.t. $z$, and $D^{-1}$ stand for the inverse operator of $D$. Consider the commutator equation of ordinary differential operators

$$
[Q, P]=1, \quad Q:=D^{q}+\sum_{k=2}^{q} w_{k} D^{q-k}, \quad P:=D^{p}+\sum_{k=2}^{p} v_{k} D^{p-k}
$$

for a couple of positive integers $(q, p)$. The above equation is called the string Equation (or Douglas equation) of type $(q, p)$, and appears in the string theory or the theory of 2D quantum gravity [1]-[8]. In the followings, we set $(q, p)=(2,2 n+1)$ for a positive integer $n$.

In the case where $(q, p)=(2,3)$, the string equation is written as an ODE satisfied by the potential $w$ of Sturm-Liouville operator $Q=D^{2}+w$, and then, by a fractional linear transformation, it is reduced to the first Painlevé equation [9]-[11]. In fact, the string equation of type $(2,3)$

$$
[Q, P]=1, \quad Q:=D^{2}+w, \quad P:=D^{3}+v_{2} D+v_{3},
$$

i.e.

$$
\left(2 v_{2}^{\prime}-3 w^{\prime}\right) D^{2}+\left(v_{2}^{\prime \prime}+2 v_{3}^{\prime}-3 w^{\prime \prime}\right) D+\left(v_{3}^{\prime \prime}-w^{\prime \prime \prime}-v_{2} w^{\prime}\right)=1,
$$

is written as an equation $w^{\prime \prime \prime}+6 w w^{\prime}+4 C_{1} w^{\prime}+4=0$ or integrated one $w^{\prime \prime}+3 w^{2}+4 C_{1} w^{\prime}+4=0$ with integral 
constants $C_{1}$ and $C_{2}$ by putting $v_{2}=\frac{3}{2} w+C_{1}, v_{3}{ }^{\prime}=\frac{3}{4} w^{\prime \prime}$, which is reduced to the first Painlevé equation

$$
w^{\prime \prime}=6 w^{2}+z
$$

by replacement $\quad z \mapsto \alpha z+\beta, \quad w \mapsto \gamma w+\delta \quad(\alpha, \gamma \neq 0) \quad$ with $\quad \alpha=2^{-1 / 5}, \quad \gamma=-4 \alpha^{3}, \quad \beta=C_{1}^{2} / 3-C_{2}$, $\delta=-2 C_{1} / 3$.

In the case where $(q, p)=(2,5)$, the string equation

$$
[Q, P]=1, \quad Q:=D^{2}+w, \quad P:=D^{5}+v_{2} D^{3}+v_{3} D^{2}+v_{4} D+v_{5}
$$

is similarly reduced to

$$
w^{(4)}=20 w^{\prime \prime} w+10 w^{\prime 2}-40 w^{3}-8 a w-\frac{8}{3} z
$$

by replacement $z \mapsto \alpha z+\beta, w \mapsto \gamma w+\delta \quad(\alpha, \gamma \neq 0)$ with $\alpha=-3^{-1 / 7}, \gamma=6 \alpha^{5}$ and suitable $\beta, \delta$, $a$. That is the 4 th order equation of the first Painlevé hierarchy.

\subsection{The First Painlevé Hierarchy}

Now we recall the definition of the first Painlevé hierarchy. Consider the serial equations

$$
d_{n+1}[w] / 4+z=0
$$

for $n \in \mathbb{N}$, where $d_{n}[w]$ is an expression of a given meromorphic function $w$ defined by $d_{0}[w]=1$ and $D d_{n+1}[w]=D G_{w} d_{n}[w]$ with $G_{w}:=D^{2}-8 D^{-1} w D-4 D^{-1} w^{\prime}$. The equations are derived from the singular manifold equation for the KdV hierarchy, and we call them the first Painlevé hierarchy [10] [12] [13]. For example, $d_{1}[w] / 4=-w+C_{10}$, where $C_{10}$ is an integral constant. In the followings, each $C_{i j}$ is also an integral constant.

If $n=1$, we have $d_{2}[w] / 4=-w^{\prime \prime}+6 w^{2}+C_{21} d_{1}[w]+C_{20}$, then $d_{2}[w] / 4+z=0$ essentially coinsides with the first Painlevé equation.

If $n=2$, we have $d_{3}[w] / 4=-w^{(4)}+20 w w^{\prime \prime}+10 w^{\prime 2}-40 w^{3}+C_{32} d_{2}[w]+C_{31} d_{1}[w]+C_{30}$, and then

$$
w^{(4)}=20 w w^{\prime \prime}+10 w^{\prime 2}-40 w^{3}+C_{32} d_{2}[w]+C_{31} d_{1}[w]+C_{30}+z .
$$

Again, it essentially coinsides with (5), i.e. the 4th order equation of the first Painlevé hierarchy.

As proved by K. Takasaki [8], the string equation of type $(2,2 n+1)$ is equivalent to (6). So, in this article, we also call (6) the string equation of type $(2,2 n+1)$.

Note that S. Shimomura [14] proved the theorems as follows.

Theorem A [14]. Each $d_{n+1}[w]$ is a differential polynomial of $2 n$-th order, i.e. each (6) is an ordinary differential equation of $2 n$-th order.

Theorem B [14]. At each pole $z=z_{0}$, the meromorphic solution to (6) has the form

$$
w(z)=\frac{k(k+1) / 2}{\left(z-z_{0}\right)^{2}}+O(1)
$$

for some positive integer $k=k\left(z_{0}\right) \in\{1, \ldots, n\}$.

The author proved a theorem similar to Theorem A for the second Painlevé hierarchy [15], and, in its proof, auxiliary differential polynomials play important roles. So, for the first Painlevé hierarchy as well, the auxiliary differential polynomials should exist. Recall them.

Theorem C [16]. Define $c_{n}[w]$ by $D c_{n}[w]=w D d_{n}[w]$. Then each $c_{n+1}[w]$ is a differential polynomial of $2 n$-th order.

\subsection{Autonomous Limits}

The first Painlevé equation has the autonomous limit [9]. Replacing $(w, z)$ by $\left(\varepsilon^{-2} w, \varepsilon z+\varepsilon^{-4} b\right)$ with a con- 
stant $b \in \mathbb{C}$, and taking limit $\varepsilon \rightarrow 0$, we obtain $w^{\prime \prime}=6 w^{2}+b$ which is satisfied by the Weierstrass' elliptic function, i.e.

$$
\wp(z):=\frac{1}{z^{2}}+\Sigma^{\prime}\left\{\frac{1}{\left(z-\Omega_{m, n}\right)^{2}}-\frac{1}{\Omega_{m, n}{ }^{2}}\right\},
$$

where $\Omega_{m, n}:=2 m \omega_{1}+2 n \omega_{3}$ for some $\omega_{1}, \omega_{3} \in \mathbb{C} \backslash\{0\}$ satisfying $\omega_{1} / \omega_{3} \in H:=\{z \in \mathbb{C} \mid \operatorname{Im} z>0\}$. Here $\Sigma^{\prime}$ means the sum for $(m, n) \in \mathbb{Z}^{2} \backslash\{(0,0)\}$. It is well-known that $\wp(z)$ is a doubly periodic meromorphic function with two fundamental periods $2 \omega_{1}, 2 \omega_{3} . \wp(z)$ satisfies the differential equation

$\wp^{\prime}(z)^{2}=4 \wp(z)^{3}-g_{2} \wp(z)-g_{3}$ and then

$$
\wp^{\prime \prime}(z)=6 \wp(z)^{2}-g_{2} / 2,
$$

where

$$
g_{2}:=\frac{1}{60} \Sigma^{\prime} \frac{1}{\Omega_{m, n}{ }^{4}}, \quad g_{3}:=\frac{1}{140} \Sigma^{\prime} \frac{1}{\Omega_{m, n}{ }^{6}} .
$$

For $n=2$, a similar result is valid, i.e.

Theorem D [17]. The 4th order equation of the first Painlevé hierarchy with suitable parameters

$$
w^{(4)}=20 w w^{\prime \prime}+10 w^{\prime 2}-40 w^{3}-8 a w-\frac{8}{3} z
$$

is reduced to the autonomous equation

$$
w^{(4)}=20 w w^{\prime \prime}+10 w^{\prime 2}-40 w^{3}-8 a w-\frac{8}{3} b
$$

by replacing $(w, z, a)$ by $\left(\varepsilon^{-2} w, \varepsilon z+\varepsilon^{-6} b, \varepsilon^{-4} a\right)$, and taking the limit $\varepsilon \rightarrow 0$.

Note that the Equation (8) is obtained as a section of the most degenerated 2D Garnier system [18] (see also [19] [20]). The following theorem is not trivial but natural if we consider Theorem $\mathrm{D}$ together with Theorem B.

Theorem E [17]. For suitable $(a, b) \in \mathbb{C}^{2}$, the autonomous Equation (9) has a solution concretely described as

$$
w(z)=\wp(z), 3 \wp(z)
$$

\subsection{Results}

A result similar to Theorem $\mathrm{D}$ is valid for $n \in \mathbb{N}$.

Theorem 1.1. The autonomous limit of the string equation of type $(2,2 n+1)$ is given by

$$
d_{n+1}[w] / 4+b=0 \text {, }
$$

where $b \in \mathbb{C}$ is a complex parameter.

Proof. Note that $d_{n+1}[w]$ has the form

$$
d_{n+1}[w]=[\text { terms with weight }-2(n+1)]+\sum_{0 \leq j \leq n} C_{n+1, j} d_{j}[w]
$$

with the weight [14] $\mathrm{wt}$ defined by $\operatorname{wt}(z)=1$ and $\operatorname{wt}(w)=-2$. After replacement

$$
\left(z, w,\left\{C_{n+1, j}\right\}_{0 \leq j \leq n}\right) \mapsto\left(\varepsilon z+\varepsilon^{-2(n+1)} b, \varepsilon^{-2} w,\left\{\varepsilon^{-2(n+1-j)} C_{n+1, j}\right\}_{0 \leq j \leq n}\right),
$$

taking the limit $\varepsilon \rightarrow 0$, we obtain the conclusion. $\square$

For the autonomous limit Equation (10), each auxiliary differential polynomial obtained in Theorem C has clear meaning.

Theorem 1.2. The differential polynomial $c_{n+1}[w]$ is the first integral of (10).

Proof. By definition, $c_{n+1}[w]=D^{-1} w D d_{n+1}[w]=w d_{n+1}[w]-D^{-1} w^{\prime} d_{n+1}[w]$. Using this relation together with the 
equation $d_{n+1}[w] / 4+b=0$, we obtain $c_{n+1}[w]=$ const. $\quad$ 口

Now we extend Theorem $E$ to the case where

Theorem 1.3. Weierstrass' elliptic function $\wp(\mathrm{z})$ is a solution to (10) with suitable parameters.

Moreover, we can prove the theorem as follows:

Theorem 1.4. For each integer $k$ satisfying $1 \leq k \leq n$,

$$
w(z)=\frac{k(k+1)}{2} \wp(z)
$$

is a solution to (10) with suitable parameters.

The proofs of these two theorems are given in the next section.

\section{Proofs of Theorem 1.3 and 1.4}

\subsection{Proof of Theorem 1.3}

Let all of $C_{n+1, j}$ 's for $1 \leq j \leq n$ vanish. Take $w$ satisfying

$$
d_{2}[w] / 4=-w^{\prime \prime}+6 w^{2}=k \text { : const. }
$$

with suitable $C_{20}$, then we have

$$
\begin{array}{ll}
d_{3}[w]=G_{w} d_{2}[w] / 4=-k w, & d_{4}[w]=G_{w} d_{3}[w] / 4=k^{2}, \\
d_{5}[w]=G_{w} d_{4}[w] / 4=-k^{2} w, & d_{6}[w]=G_{w} d_{5}[w] / 4=k^{3}, \\
d_{7}[w]=G_{w} d_{6}[w] / 4=-k^{3} w, & d_{8}[w]=G_{w} d_{7}[w] / 4=k^{4}, \\
d_{9}[w]=G_{w} d_{8}[w] / 4=-k^{4} w, & d_{10}[w]=G_{w} d_{9}[w] / 4=k^{5},
\end{array}
$$

with suitable $C_{n+1,0}$ 's. Putting $k=0$, i.e. choosing $w=\wp(z)$ as it satisfies $w^{\prime \prime}=6 w^{2}$, we obtain the conclusion.

\subsection{Proof of Theorem 1.4}

Theorem 1.4 immediately follows from the following lemma.

Lemma. For every positive integer $k, G_{\frac{k(k+1)}{2} \wp} \wp^{j}(j=1, \ldots, k)$ is described by some polynomial of $\wp$, and its degree is as follows:

$$
G_{\frac{k(k+1)}{2} \wp^{\prime}} \wp^{j}=\left\{\begin{array}{lc}
O\left(\wp^{j+1}\right) & \text { for } 1 \leq j \leq k-1, \\
O\left(\wp^{j-1}\right) & \text { for } j=k .
\end{array}\right.
$$

Proof. Using $\wp^{\prime 2}=4 \wp^{3}-g_{2} \wp-g_{3}$ and $\wp^{\prime \prime}=6 \wp^{2}-g_{2} / 2$,

$$
G_{\frac{k(k+1)}{2} \wp} \wp^{j}=\left\{4 j(j-1)+6 j-4 k(k+1)\left(1-\frac{1}{2(j+1)}\right)\right\} \wp^{j+1}-j\left(j-\frac{1}{2}\right) g_{2} \wp^{j-1}-j(j-1) g_{3} \wp^{j-2}+\text { const. }
$$

So, if $j=k$, the coefficient of $\wp^{k+1}$ vanishes as follows:

$$
4 k(k-1)+6 k-4 k(k+1)\left(1-\frac{1}{2(k+1)}\right)=4 k(k-1)+6 k-4 k(k+1)+2 k=0 .
$$

Thus, we have

$$
G_{\frac{k(k+1)}{2} \wp} \wp^{k}=O\left(\wp^{k-1}\right) .
$$

Note that $d_{n+1}\left[\frac{k(k+1)}{2} \wp\right]$ is a polynomial in $\wp$ of degree $n$, and all terms but one of top degree have 
integral constants. Therefore, if the term of top degree vanishes, we can make all terms vanish with suitable selection of integral constants. Thus, Theorem 1.4 is established.

\section{Discussion}

The results of this article are summarized as follows: we obtained the autonomous limit of the string equation of type $(2,2 n+1)$ with a first integral $c_{n+1}[w]$, and gave its special solutions written by Weierstrass' elliptic function as $w(z)=\frac{k(k+1)}{2} \wp(z)$ for $1 \leq k \leq n$.

Of course, poles of these solutions are uniform, i.e. every pole $z=z_{0}$ of $w(z)=\frac{k(k+1)}{2} \wp(z)$ gives the Laurent expansion with the same dominant term $w(z)=\frac{k(k+1) / 2}{\left(z-z_{0}\right)^{2}}+O(1)$. However, the possibility of the existence of solution without uniformity is not excluded. Even two types of poles allow us infinitely many patterns. So, we have problems on the patterns of poles. Can we construct elliptic function solutions to the autonomous limit of the string equation of type $(2,5)$ (or type $(2,2 n+1)$ for $n \geq 2)$ with both types of pole $\frac{1}{\left(z-z_{0}\right)^{2}}+O(1)$ and $\frac{3}{\left(z-z_{0}\right)^{2}}+O(1)$ ? Is any distribution of the two (or more) kinds of poles admitted? If not, how many or what kind of patterns are admitted?

Another remark should be given. T. Oshima and H. Sekiguchi [21] studied the commutator equation $[Q, P]=0$ of partial differential operators $Q, P$ invariant under the action of a Weyl group, and obtained many of elliptic function solutions. Note that the autonomous limit of $[Q, P]=1$ means $[Q, P]=0$. The fact implies that, in view of the string theory, the first Painlevé equation is not only a nonautonomization but also a quantization of the Weierstrass' elliptic function. Relation between their solutions and our special solutions should be studied in the future. It may yield a new kind of quantization of KdV equation or hierarchy. Autonomous limit is a kind of approximation of the differential equation. Therefore, the solutions of the autonomous limit equation gives us information on the asymptotics of the nonautonomous equation, as well as does on the first Painlevé equation. Moreover, if all of the solutions to the autonomous limit equation are determined, it contributes the argument on the irreducibility of the string equation in the sence of the differential Galois theory, as well as on the irreduciblity of the first Painlevé equation.

\section{Acknowledgements}

The author wishes to acknowledge Prof. T. Oshima for his helpful comment.

\section{References}

[1] Adler, M. and van Moerbeke, P. (1992) A Matrix Integral Solution to Two-Dimensional $W_{p}$-Gravity. Comm. Math. Phys., 147, 25-26. http://dx.doi.org/10.1007/BF02099527

[2] Douglas, M.R. (1990) String in Less than One-Dimensions and KdV Hierarchies. Phys. Lett. B, 238, 176-180. http://dx.doi.org/10.1016/0370-2693(90)91716-O

[3] Fukuma, M., Kawai, H. and Nakayama, R. (1991) Infinite Dimensional Grassmannian Structure of Two Dimensional String Theory. Comm. Math. Phys., 143, 371-403. http://dx.doi.org/10.1007/BF02099014

[4] Kac, V. and Schwarz, A. (1991) Geometric Interpretation of Partition Functions of 2D Gravity. Phys. Lett. B, 257, 329334. http://dx.doi.org/10.1016/0370-2693(91)91901-7

[5] vanMoerbeke, P. (1994) Integrable Foudations of String Theory. In: Babelon, O., et al., Eds., Lectures on Integrable Systems, World Sci. Publ., Singapore, River Edge, 163-267.

[6] Moore, G. (1990) Geometry of the String Equations. Comm. Math. Phys., 133, 261-304. Matrix Models of 2D Gravity and Isomonodromic Deformations. Prog. Theor. Phys. Suppl., 102, 255-285. http://dx.doi.org/10.1143/PTPS.102.255

[7] Schwarz, A. (1991) On Solutions to the String Equations. Mod. Phys. Lett. A, 29, 2713-2725. http://dx.doi.org/10.1142/S0217732391003171 
[8] Takasaki, K. (2007) Hamiltonian Structure of PI Hierarchy. SIGMA, 3, 42-116. http://dx.doi.org/10.3842/sigma.2007.042

[9] Conte, R. and Mussette, M. (2008) The Painlevé Handbook. Springer Science + Business Media B.V., Dordrecht.

[10] Gromak, V.I., Laine, I. and Shimomura, S. (2002) Painlevé Differential Equations in the Complex Plane. Walter de Gruyter, Berlin, New York. http://dx.doi.org/10.1515/9783110198096

[11] Ince, E.L. (1956) Ordinary Differential Equations. Dover Publ., New York.

[12] Kudryashov, N.A. (1997) The First and Second Painlevé Equations of Higher Order and Some Relations between Them. Phys. Lett. A, 224, 353-360. http://dx.doi.org/10.1016/S0375-9601(96)00795-5

[13] Weiss, J. (1984) On Classes of Integrable Systems and the Painlevé Property. J. Math. Phys., 25, 13-24. http://dx.doi.org/10.1063/1.526009

[14] Shimomura, S. (2004) Poles and $\alpha$-Points of Meromorphic Solutions of the First Painlevé Hierarchy. Publ. RIMS, Kyoto Univ., 40, 471-485. http://dx.doi.org/10.2977/prims/1145475811

[15] Sasaki, Y. (2013) Lower Estimates of the Growth Order of Solutions to the Second Painlevé Hierarchy. J. Math. Phys., 54, 073510

[16] Sasaki, Y. (2015) Auxiliary Differential Polynomials for the First Painlevé Hierarchy. Proc. Japan Acad., Ser. A, 91, 7-8. http://dx.doi.org/10.3792/pjaa.91.7

[17] Sasaki, Y. (2014) Weierstrass’ Elliptic Function Solution to the Autonomous Limit of the String Equation of Type (2,5). Adv. Pure Math., 4, 494-497. Erratum to "Weierstrass' Elliptic Function Solution to the Autonomous Limit of the String Equation of Type (2,5)”. [Advances in Pure Mathematics 4 (2014), 494-497], ibid., 680-681.

[18] Shimomura, S. (2000) Painlevé Property of a Degenerate Garnier System of (9/2)-Type and a Certain Fourth Order Non-Linear Ordinary Differential Equation. Ann Scuola Norm. Sup. Pisa Cl. Sci., XXIX, 1-17.

[19] Kimura, H. (1989) The Degeneration of the Two Dimensional Garnier System and the Polynomial Hamiltonian Structure. Ann.Mat. Pura. Appl., 155, 25-74. http://dx.doi.org/10.1007/BF01765933

[20] Suzuki, M. (2006) Spaces of Initial Conditions of Garnier System and Its Degenerate Systems in Two Variables. J. Math. Soc. Japan, 58, 1079-1117. http://dx.doi.org/10.2969/jmsj/1179759538

[21] Oshima, T. and Sekiguchi, H. (1995) Commuting Families of Differential Operators Invariant under the Action of a Weyl Group. J. Math. Sci. Univ. Tokyo, 2, 1-75. 